\title{
Determinants of delay in seeking treatment among malaria patients in Dera district, NorthWest Ethiopia: a case control study
}

\author{
Melkamu Tiruneh ${ }^{1}$, Gebremedhin Berhe Gebregergs ${ }^{2,3}$, Dereje Birhanu ${ }^{3}$
}

1. Malaria Control and Elimination Partnership in Africa (MACEPA) - PATH: A Catalyst for Global Health, Finote Selam, Ethiopia

2.School of Public Health, College of Health Sciences, Mekelle University, Mekelle, Ethiopia

3.School of Public Health, College of Medicine and Health Sciences, Bahir Dar University, Bahir Dar, Ethiopia

\begin{abstract}
Background: Prompt and effective treatment of malaria is critical because delays increase the risk for serious illness, disability and death.

Objective: To assess determinants of delay in seeking treatment among malaria patients at Dera district, NorthWest Ethiopia. Methods: A case control study was conducted from September 01 to October 15, 2014. A total of 318 malaria patients diagnosed using microscopy or rapid diagnostic test, and who sought treatment in health centers were interviewed. Multivariable logistic regression was done to identify determinants of delay.

Results: Delay was high when a patient earned less than 25.0 USD [AOR=15.7, 95\% CI: 4.8 - 51.2] and 9.6 times higher if he/ she was not a member of community based health insurance [AOR=9.6, 95\% CI: $4.4-21.3$ ]. Respondents who travelled for more than 30 minutes to get to a health facility $[\mathrm{AOR}=4.4,95 \% \mathrm{CI}: 1.2-15.9]$ were more likely to be late in seeking treatment for malaria.

Conclusion: Income, community based health insurance, previous history of malaria infection, decision making and distance were determinants of delay in seeking treatment for malaria. To reduce the delay, interventions should focus on outreach malaria services and increase enrollment to community based health insurance.
\end{abstract}

Keywords: Malaria; treatment seeking; Ethiopia.

DOI: https://dx.doi.org/10.4314/ahs.v18i3.12

Cite as: Tiruneh M, Gebregergs GB, Birhanu D. Determinants of delay in seeking treatment among malaria patients in Dera district, Northwest Ethiopia: a case control study. Afri Health Sci. 2018;18(3): 552-559. https://dx.doi.org/10.4314/abs.v18i3.12

\section{Introduction}

Malaria is one of the top leading causes of morbidity and mortality in the developing world. The burden is heaviest in the World Health Organization (WHO) African Region, where an estimated $90 \%$ of all malaria deaths oc-

\section{Corresponding author:}

Gebremedhin Berhe Gebregergs,

Mekelle University, Mekelle, Ethiopia

P.O.Box: 1871

Telephone number +251912761885

Fax number +251344416681

E-Mail:bgebrish04@gmail.com cur ${ }^{1}$. It also accounts for $2.6 \%$ and $10.8 \%$ of disability adjusted life years (DALYs) in the world and in sub-Saharan Africa, respectively ${ }^{2}$.

In Ethiopia, about $68 \%$ of the population live in malaria endemic areas, and $75 \%$ of the land mass of the country is at risk of malaria. A total of 3,384,589 malaria cases were reported in 2012 with $80 \%$ completeness of reporting health facilities. However, the actual number of malaria cases was estimated to be higher ${ }^{3,4}$. Nationally, malaria accounted $17 \%$ and $18 \%$ of morbidity in adults and under five children, respectively. It is the second (8\%) cause of admission following pneumonia, and number one, $(9 \%)$, cause of mortality ${ }^{5}$.

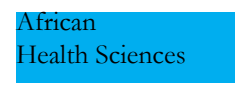

(C) 2018 Tiruneh et al. Licensee African Health Sciences. This is an Open Access article distributed under the terms of the Creative commons Attribution License (https://creativecommons.org/licenses/BY/4.0), which permits unrestricted use, distribution, and reproduction in any medium, provided the original work is properly cited. 
In Amhara region, $22 \%$ of out-patient visits, $24 \%$ of hospital admissions and $10 \%$ of health facility deaths were due to malaria ${ }^{6}$. In Dera, the study setting, malaria is the top leading cause of morbidity in adults and under-five children; accounting for $40 \%$ and $42 \%$, respectively. Dera is found in Amhara region, Ethiopia. There were 11 health centers, 32 health posts (public) and five private clinics. Malaria diagnosis and treatment is given based on result of either Rapid Diagnostic Test (RDT) or microscopy at the public health facilities. There was no malaria RDT at all the private health facilities ${ }^{7}$.

Prompt parasitological confirmation within 24 hours onset of fever by microscopy or Rapid Diagnostic Test (RDT) is recommended for all patients suspected of malaria before the initiation of treatment. Treatment solely on the basis of clinical suspicion is considered only if parasitological diagnosis is not available on $\operatorname{spot}^{8,9}$. In Ethiopia, malaria treatment with only Artemisinin-based Combination Therapy is free at all levels of the public health facilities while the other anti-malarial drugs are not. Diagnosis at health post (service delivered by the community health workers) is free, and the tool is malaria rapid diagnostic test (RDT). However, diagnosis at the health center and above is not free and the diagnostic tool is microscopy.

In unstable malaria transmission, the disease can progress from mild to severe stage, and unacceptable death within 24 hours after the onset of fever might happen if it is left untreated. The Ethiopian malaria prevention, control and elimination strategy gives more emphasis to early diagnosis and treatment with effective anti-malaria drugs within 24 hours of onset of fever to decrease the risk for serious illness, disability, and death ${ }^{3,10}$. Ethiopia has been implementing Community-based Health Insurance (CBHI) schemes since 2011. It is an alternative to user fees to improve equity in access to medical care particularly to those rural communities and the informal sector. It has the potential to increase utilization, better protect people against (catastrophic) health expenses and address issues of equity ${ }^{11}$. Nevertheless, only $10.3 \%$ of malaria patients in the study area seek treatment within the standard ${ }^{7}$. Hence, the present study was developed to identify determinants of delay in seeking treatment among malaria patients in Dera district, NorthWest Ethiopia.

\section{Methods \\ Study design and setting}

A case control study was conducted in Dera district, NorthWest Ethiopia from September 01 to October 15, 2014. It was conducted in eleven public health centers. The district is a malaria endemic area with a prevalence of $19.4 \%$, and it experiences seasonal malaria transmission with peak from September to December. A total of 54,295 malaria cases were diagnosed and treated in 2013, and the annual parasite index was estimated to be 194 per 1000 population $^{7}$.

\section{Study population}

The study population consisted of cases and controls. Cases were patients who had one or more signs/symptoms of malaria and positive for Plasmodium species based on microscopy or rapid malaria diagnostic test, and sought treatment in the health centers after 24 hours of developing the first signs/symptoms. Controls were patients who had one or more signs/symptoms of malaria and positive for Plasmodium species based on microscopy or rapid diagnostic test, and sought treatment in the health centers within 24 hours of developing the first signs/symptoms.

\section{Sample size and sampling technique}

Sample size was calculated using Epi-Info version 7.0 (Center for Disease Control and Prevention, Atlanta, GA, USA). We assumed a confidence interval of $95 \%$, power of $80 \%$ and case to control ratio of $1: 1$. From other study, we used odds ratio of 4.96 , and proportion of individuals who fear drug side effects was $2.6 \%$ among controls ${ }^{12}$. By adding $10 \%$ none response rate, the total sample size was 322 (161 cases and 161 controls). Cases were selected using systematic random sampling technique. For a selected case, a consecutive control was enrolled from the same health center.

\section{Data collection procedure and tool}

Cases and controls were interviewed by trained health workers using structured and pre-tested questionnaire. The tool was initially prepared in English, and translated to Amharic, local language. The questionnaire encompassed socio-demographic and economic variables, knowledge and perception about malaria, awareness, treatment seeking behavior and decision making profile of respondents. 


\section{Operational definition}

Sufficient knowledge: participants who score $70 \%$ or more of the knowledge questions related to the signs and symptoms, mode of transmission, prevention and treatment of malaria.

Perceived severity: One's perception of how serious a condition might be and its medical or social consequences.

\section{Data processing and analysis}

Data were entered, cleaned, coded and analyzed using SPSS version 16.0. OR with 95\% CI was used to measure association. Variables (sex, residence, religion, educational status, distance (minutes), knowledge, decision making, member of CBHI, monthly income, and ever been infected) which showed association at $\mathrm{p}<0.2$ in the bivariate analysis were fitted to the multivariate binary logistic regression model. P-value $<0.05$ in multivariate binary logistic regression was considered as statistically significant.

\section{Ethical considerations}

Ethical clearance was obtained from the Ethical Review Committee of Bahir Dar University after reviewing the research protocol including ethical procedures. Written permission was obtained from Amhara Regional Health Bureau and Dera district health office. Informed oral consent was obtained from respondents as majority of them were unable to read and write. Enumerators explained the study objective to all participants. All the information obtained from the respondents was treated as private and confidential. Cases and controls were treated based on the national treatment guidelines.

\section{Results}

\section{Socio-demographic characteristics}

A total of 318 participants were interviewed (159 cases and 159 controls). The mean age of patients was 30.3 (SD \pm 12.0$)$. More than three fourth $(77.4 \%)$ of the respondents were males and majority $(90.3 \%)$ of the participants were from rural. Orthodox Christian was the predominant religion $(84.6 \%)$.

Over half of the participants (57.2\%) were unable to read and write. Eighty two percent of the cases and nearly two third $(64.2 \%)$ of the controls were currently married. Concerning their occupation, $221(69.5 \%)$ were farmers, $59(18.6 \%)$ were students, $18(5.7 \%)$ were merchants and the remaining $(6.2 \%)$ were housewives, daily laborers or government employees.

The median monthly income of respondents was 500.0 ET Birr (25.0 USD) with inter quartile range of 200.0 ET Birr (10.0 USD) for cases and 900.0 ET Birr (45.0 USD) with inter quartile range of $650.0 \mathrm{ET}$ Birr (32.5 USD) for controls.

One hundred fifty two cases (95.6\%) and 96 (60.4\%) controls took more than 30 minutes to get to a nearby health facility. Few of the cases $(14.5 \%)$ and three fourth (76.1\%) of controls were members of community based health insurance (Table 1). 


\section{Table 1. Socio - demographic characteristics of respondents in Dera district, Ethiopia, 2014.}

\begin{tabular}{llll}
\hline Variables & Level & \multicolumn{2}{l}{ Patient Categories } \\
\cline { 2 - 4 } & & Cases & Controls \\
\hline Sex & Male & $131(82.4 \%)$ & $115(72.3 \%)$ \\
Religion & Female & $28(17.6 \%)$ & $44(27.7 \%)$ \\
& Orthodox Christian & $143(89.9 \%)$ & $126(79.2 \%)$ \\
Age & Muslim & $16(10.1 \%)$ & $33(20.8 \%)$ \\
& $<5$ & $3(1.9 \%)$ & $1(0.6 \%)$ \\
Marital status & $5-14$ & $2(1.3 \%)$ & $22(13.8 \%)$ \\
& $\geq 15$ & $154(96.9 \%)$ & $136(85.5 \%)$ \\
\multirow{2}{*}{ Educational status } & Married & $130(81.8 \%)$ & $102(64.2 \%)$ \\
& Never married & $24(15.1 \%)$ & $53(33.3)$ \\
& DWS* & $5(3.1 \%)$ & $4(2.5 \%)$ \\
& Unable to read & $103(64.8 \%)$ & $79(49.7 \%)$ \\
& write & & \\
& Primary school & $41(25.8 \%)$ & $47(29.6 \%)$ \\
Occupation & Secondary school & $11(6.9 \%)$ & $23(14.5 \%)$ \\
& Above secondary & $4(2.5 \%)$ & $10(6.3 \%)$ \\
& Student & $16(10.1 \%)$ & $43(27.0 \%)$ \\
& Farmer & $130(81.8 \%)$ & $91(57.2 \%)$ \\
Member of CBHI & Merchant & $6(3.8 \%)$ & $12(7.5 \%)$ \\
& Housewife & $1(0.6 \%)$ & $4(2.5 \%)$ \\
& Others* & $6(3.8 \%)$ & $9(5.7 \%)$ \\
& $\leq 30$ minutes & $7(4.4 \%)$ & $63(39.6 \%)$ \\
& $>30$ minutes & $152(95.6 \%)$ & $96(60.4 \%)$ \\
& Yes & $23(14.5 \%)$ & $121(76.1 \%)$ \\
& No & $136(85.5 \%)$ & $38(23.9 \%)$ \\
\hline
\end{tabular}

Others*- Housewife, daily laborer or government employee; DWS*- Divorced/Widowed/Separated; CBHI - Community Based Health Insurancє

Knowledge and perception of respondents

One third of cases $(35.2 \%)$ and half of the controls $(54.7 \%)$ had sufficient knowledge on malaria. Majority $(85.5 \%)$ of the cases and almost all $(98.7 \%)$ controls perceived malaria as a serious and killer disease. One in ten cases $(91.8 \%)$ had ever been infected with malaria prior to the current illness. Majority of the cases $(93.1 \%)$ and controls (96.9\%) had ever seen/ heard message about malaria. Decision to seek treatment was made by the patient him/herself in $14 \%$ and $74 \%$ of the cases and controls, respectively (Table 2 ). 
Table 2. Knowledge, perception and awareness of participants about malaria in Dera district, Ethiopia, 2014.

\begin{tabular}{llll}
\hline Variables & Level & \multicolumn{2}{l}{ Patient Categories } \\
\cline { 3 - 4 } & & Cases & Controls \\
\hline Knowledge about malaria & Sufficient & $76(47.8 \%)$ & $102(64.2 \%)$ \\
& Insufficient & $83(52.2 \%)$ & $57(35.8 \%)$ \\
Vulnerable to acquire malaria & Yes & $116(73 \%)$ & $104(65.4 \%)$ \\
& No & $43(27.0 \%)$ & $55(34.6 \%)$ \\
Malaria is Serious \& Killer & Yes & $136(85.5 \%)$ & $157(98.7)$ \\
& No & $23(14.5 \%)$ & $2(1.3 \%)$ \\
Ever been infected by malaria & Yes & $146(91.8 \%)$ & $61(38.4 \%)$ \\
& No & $13(8.2 \%)$ & $98(61.6 \%)$ \\
Ever heard about malaria & Yes & $148(93.1 \%)$ & $154(96.9 \%)$ \\
& No & $11(6.9 \%)$ & $5(3.1 \%)$ \\
Decision & Head of household & $137(86.2 \%)$ & $41(25.8 \%)$ \\
& Patient him/herself & $22(13.8 \%)$ & $118(74.2 \%)$
\end{tabular}

\section{Reasons of delay among cases}

Shortage of time $(17.0 \%)$, inconvenient service delivery time $(12.6 \%)$, fear of treatment cost $(9.1 \%)$, transport inaccessibility $(6.6 \%)$ and being a mild illness (4.4\%) were reasons mentioned by the malaria patients who came after 24 hours of onset of sign/symptom.

\section{Determinants of delay in seeking treatment}

In multivariate logistic regression (adjusted analysis), average monthly income, community based health insurance, history of malaria infection, decision making and distance were independently associated with delay in seeking treatment among malaria patients in the setting. This study revealed that age, sex, residence, religion, perceived severity, knowledge about malaria and educational status were not associated with delay in seeking treatment.

Participants in low monthly income category $[\mathrm{AOR}=15.7$, 95\% CI: 4.8 - 51.2] and who were not members of community based health insurance $[\mathrm{AOR}=9.6,95 \% \mathrm{CI}: 4.5$ - 21.3] were more likely to be late in seeking treatment for malaria. Patients whose decision, to seek treatment, was made by head of household were six times $[\mathrm{AOR}=6.30$, 95\% CI: 2.8 -14.2] more likely to delay compared with patients who decided by themselves. Distance: as the time patients spent to get to a health facility increases, the pattern of delay increases $[\mathrm{AOR}=4.4,95 \% \mathrm{CI}: 1.2-15.2]$ (Table 3). 
Table 3 Variables associated with delay in malaria treatment seeking at Dera district, Ethiopia, 2014.

\begin{tabular}{|c|c|c|c|c|c|}
\hline Variables & & \multicolumn{2}{|l|}{$\begin{array}{l}\text { Delay } \\
\text { treatment }\end{array}$} & COR (95\% CI) & $\operatorname{AOR}(95 \%$ CI) \\
\hline \multirow{2}{*}{ Sex } & Male & 131 & 115 & $1.8(1.1,3.1)$ & $10(0.428)$ \\
\hline & Female & 28 & 44 & 1.0 & 1.0 \\
\hline \multirow[t]{2}{*}{ Distance(minutes) } & $\leq 30$ Minutes & 7 & 63 & 1.0 & 1.0 \\
\hline & > 30 Minutes & 152 & 96 & $14.3(6.2,32.4)$ & $4.4(1.2,15.2)^{*}$ \\
\hline \multirow[t]{2}{*}{ Knowledge } & Sufficient & 76 & 102 & 1.0 & 1.0 \\
\hline & Insufficient & 83 & 57 & $2.0(1.3,3.1)$ & $1.0(0.4,2.2)$ \\
\hline \multirow[t]{2}{*}{ Decision making } & Household head & 137 & 41 & $17.9(10.1,31.8)$ & $6.3(2.8,14.1)^{*}$ \\
\hline & Patient & 22 & 118 & 1.0 & 1.0 \\
\hline \multirow[t]{2}{*}{ Member of CBHI } & Yes & 23 & 121 & 1.0 & 1.0 \\
\hline & No & 136 & 38 & $18.8(10.6,33.4)$ & $9.6(4.4,21.3)^{*}$ \\
\hline \multirow[t]{3}{*}{ Monthly income } & $\leq 25.0$ USD & 99 & 27 & $34.8(15.0,81.0)$ & $15.7(4.8,51.2)^{*}$ \\
\hline & 25.1- 49.9 USD & 52 & 56 & $8.82(3.88,20.04)$ & $4.5(1.5,13.8)^{*}$ \\
\hline & $\geq 50.0$ USD & 8 & 76 & 1.0 & 1.0 \\
\hline Ever been infected & Yes & 146 & 61 & $18.0(9.4,34.6)$ & $6.8(2.6,17.8)^{*}$ \\
\hline No & 13 & 98 & 1.0 & 1.0 & \\
\hline \multirow[t]{2}{*}{ Perceived Severity } & Yes & 136 & 157 & 1.0 & 1.0 \\
\hline & No & 23 & 2 & $13.3(3.1,57.3)$ & $2.4(0.3,20.0)$ \\
\hline
\end{tabular}

*p- value less than 0.05; CBHI - Community Based Health Insurance

\section{Discussion}

Early treatment seeking can be affected by many factors such as individual's socio economic and cognitive factors, and the health institutions that deliver the service ${ }^{13}$.

In the present study, delay in seeking treatment for malaria was significantly associated with monthly income. Accordingly, patients who earned less than 25.0 USD were sixteen times more likely to delay as compared to those who had monthly income greater than 50.0 USD. This is similar with study findings reported in Southern Ethiopia and SouthEast Asia ${ }^{14,15}$. Patients in a low income category might not afford cost of diagnosis and treatment, and may initially prefer home care and cultural remedies. Literatures cited that behavioral factors such as strategies for self-appraisal and techniques for coping with illness, emotional reactions and negative imagery elicited by the illness threat could affect treatment seeking ${ }^{16}$. Inconvenient service delivery time $(12.6 \%)$ should be considered as poor individuals may give priority to income generating activities. Besides, Study sample individuals were mostly $(69.5 \%)$ farmers. Similar finding was reported from Southern Ethiopia that caregivers preferred home treatment as they may not get time for health-seeking at formal health services ${ }^{17}$.

Community based health insurance played a capital role in early treatment seeking; participants who were not members of community based health insurance were ten times more likely to be late in seeking treatment as compared to those who were members of community based health insurance. Health insurance might avoid challenges of the payment process of health facilities in times of patient visits. In support of this, a study from Ghana affirmed that health insurance generally insulates subscribers from monetary payments which in itself increased both attendance and utilization of health services ${ }^{15}$. 
Decision making was found to be strongly associated with delay in seeking treatment. The more the decision is made by the head of household, the more a patient will be late to visit health facility. Jean-Wei CSU Q-ZX in $2012^{14}$ reported similar findings, and in Tanzania, lack of collective decision-making led to delayed care-seeking ${ }^{19}$.

Participants who had previous history of malaria infection were late in seeking treatment. While $4.4 \%$ respondents perceived malaria as a mild disease that can heal by itself, nevertheless patients might have faced financial problems if they were frequently affected with malaria. Moreover, treatment seeking maybe delayed due to failure to suspect malaria in individuals who have had another repeated febrile illness (that they assumed easy and mild). Even in situations in which the cause of the fever was believed to be malaria, people still perceived the illness as mild ${ }^{20}$.

Patients who travelled more than 30 minutes walking distance to a nearby health facility were four times more likely to be late in seeking treatment as compared to their counter parts. The finding was parallel to studies done in SouthWest Ethiopia, Myanmar and India ${ }^{12,14,21}$.This could be due to the inaccessibility of health facility to patients from remote areas.

In this study, sex of patients was not associated with delay in seeking treatment for malaria. Similar finding was documented elsewhere in Ethiopia ${ }^{12}$. However, a study done in Kenya showed that boys were more likely to get early diagnosis and treatment of malaria than girls ${ }^{22}$. The difference in study population (children vs. adults) may bring variation.

Knowledge about malaria was not significantly associated with delay in seeking treatment. This result contrasts findings from other districts of Ethiopia where knowledge plays a major role ${ }^{23}$. The sample size and study population could be a possible reason of disparity.

\section{Limitation of the study}

Misclassification of cases and controls might occur as there was no bio-marker in the blood film. Subjects' response might have been varied in different malaria seasons.

\section{Conclusion}

Delay in seeking treatment for malaria was significant- ly associated with monthly income, community based health insurance, history of malaria infection, decision making and distance. Thus, expanding the enrollment of community based health insurance; strengthening malaria outreach services and service accessibility are recommended.

\section{Acknowledgements}

We are grateful to the study participants for their cooperation during data collection.

\section{Conflict of interest}

The authors declare that they have no competing interests.

\section{References}

1. World Health Organization (WHO). World malaria report. Geneva: WHO; 2015

2. World Bank. World Development Report. New York: Oxford University Press; 1993.

3. Federal Ministry of Health. Strategic Plan for Malaria Control, Prevention and Elimination in Ethiopia, 20112015. Addis Ababa: FMOH; 2010.

4. Federal Ministry of Health. Health Sector Development Program-IV Annual Performance Report. Version I. Addis Ababa; 2012.

5. Federal Ministry of Health. Health and Health Related Indicators. Addis Ababa: Federal ministry of Health policy planning Directorate; 2011.

6. Amhara Regional Health Bureau. Public Health Emergency Management Annual Report. Babir Dar, 2012.

7. Dera district health office. Annual Performance Report. Anbessamie; 2013

8. World Health Organization (WHO). Guideline for the Treatment of Malaria. Second ed. Geneva: WHO; 2010. 9. Federal Ministry of Health. National Malaria Guidelines. Third ed. Addis Ababa, Ethiopia: FMOH; 2012 10. World Health Organization (WHO). World Malaria Report. Geneva: WHO; 2011.

11. Ethiopian public health institute. Improving Health Care Financing in Ethiopia. Addis Ababa, 2014

12. Getahun A, Deribe K, Deribew A. Determinants of delay in malaria treatment seeking behavior for under-five children in South-West Ethiopia: a case control study. Malaria Journal. 2010; 9:320.

13. Dillip A, Hetzel MW, Gosoniu D, et al. Socio-cultural 
factors explaining timely and appropriate use of health facilities for degedege in South-Eastern Tanzania. Malar J. 2009; 8: 144

14. Jian-Wei Xu Q-ZX, Hui Liu and Yi-Rou Zeng. Malaria treatment-seeking behavior and related factors of Wa ethnic minority in Myanmar: a cross-sectional study. Malaria Journal. 2012; 11(1).

15. Turuse EA, Alemu K, Beyen TK. Determinants of Delay in Malaria Prompt Diagnosis and Timely Treatment among Under-five Children in Shashogo Woreda, Hadiya Zone, Southern Ethiopia: A Case Control Study. Health. 2014; 6:950-9 PubMed .

16. Safer MA, Tharps QJ, Jackson TC, Leventhal H. Determinants of three stages of delay in seeking care at a medical clinic. Med Care. 1979 Jan; 17(1):11-29.

17. Yewhalaw D, Kassahun W, Woldemichael K, Tushune K, Sudaker M, et al. The influence of the Gilgel-Gibe hydroelectric dam in Ethiopia on caregivers' knowledge, perceptions and health-seeking behaviour towards childhood malaria. Malar J. 2010; 9: 47.

18. Zakaria HB, Asante FA. Economic Cost of Malaria Treatment under the Health Insurance Scheme in the
Savelugu-Nanton District of Ghana. Ghana Journal of Development Studies. 2013;10(1-2)

19. Kassile T, Lokina R, Mujinja P, Mmbando PB: Determinants of delay in care seeking among children under five with fever in Dodoma region, central Tanzania: a cross-sectional study. Malaria Journal. 2014 13:348.

20. Iwelunmor J, Idris O, Adelakun A, Airhihenbuwa CO: Child malaria treatment decisions by mothers of children less than five years of age attending an outpatient clinic in South-West Nigeria: an application of the PEN-3 cultural model. Malar J. 2010, 9:354.

21. Ashis Das TSR. Factors affecting treatment-seeking for febrile illness in a malaria endemic block in Boudh district, Orissa, India: policy implementations for malaria control. Malaria Journal. 2010; 9:377.

22. Taffa N, Chepngeno G. Determinants of health care seeking for childhood illnesses in Nairobi slums. Tropical Medicine and International Health. 2005; 10(3):240-245

23. Paulander J, Olsson H, Lemma H, Getachew A and Sebastian M. Knowledge, attitudes and practice about malaria in rural Tigray, Ethiopia. Global Health Action. 2009; DOI: 10.3402/gha.v2i0.1839 